\title{
INFLUENCE OF MOLECULAR ELECTRIC FIELDS ON ELASTIC PROPERTIES OF PEROVSKITE-LIKE SUBSTRATES
}

\author{
T.J. IIOFFMANN \\ Institute of Applied Mechanics, Poznań University of Technology \\ Piotrowo 3, 60-965 Poznań, Poland \\ M. Drozdowski and D. Kasprowicz \\ Institute of Physics, Poznan University of Technology \\ Piotrowo 3, 60-965 Poznań, Poland
}

\begin{abstract}
The dynamics of $\mathrm{ABCO}_{4}$ deformable crystals with electroelastic interacting was expressed by theoretical model with wave-linear approximation. The one-dimensional problem was considered assuming that the field variables are represented by plane electroelastic waves. As a result the dispersion equation for these crystals was obtained. Using this model the data concerning the elastic properties of $\mathrm{SrLaGaO}_{4}$ are discussed.
\end{abstract}

PACS numbers: $46.30 . \mathrm{Cn}$

\section{Introduction}

Some of the crystals belonging to the group of compounds with perovskitelike structure are interesting as substrates for high temperature superconducting thin films from the standpoint of lattice matching and their elastic properties $[1,2]$. Good quality of epitaxial layers requires both crystallographic perfection and appropriate physical properties of substrate materials. Owing to its simplicity the study of phenomenological models constitutes that phase of constructing the theory which enables the understanding of the fundamental relations between effects, the planning of an experiment and the choice of the microscopic model. The theory of coupled fields in which the continuum model is used belongs to this phenomenological type and covers electromagnetic, mechanical, thermal and diffusion effects. The subject of the present considerations will be elastic waves propagation in anisotropic continuum with dielectric polarization.

Using this model the data concerning the elastic properties of $\mathrm{SrLaGaO}_{4}$ single crystals belonging to the family of compounds with the general formula $\mathrm{ABCO}_{4}$, where $\mathrm{A}=\mathrm{Ca}$ or $\mathrm{Sr}, \mathrm{B}=\mathrm{Y}, \mathrm{La}$ and $\mathrm{C}=\mathrm{Al}, \mathrm{Ga}$ or some transition element, are discussed. 


\section{Theoretical model}

Let us consider infinite dielectric, deformable-elastic continuum, modelling $\mathrm{ABCO}_{4}$ lattice in the primary-molecular electric field. In this case the equations of motion are as follows [3]:

$$
\rho \ddot{u}_{k}=C_{k l m n} \partial_{l} \partial_{m} u_{n}+P_{l} \partial_{l} E_{k}
$$

where $\rho$ - density of mass, $u_{k}-$ displacement vector, $\ddot{u}_{k}$ - local acceleration, $C_{k l m n}$ - tensor of elastic constants, $\partial_{l}$ - space derivative, $P_{l}$ - electric polarization vector, $E_{k}$ - electric field intensity vector.

The equations of electrodynamics being assumed in the Maxwell form $[4,5]$ :

$$
\begin{aligned}
& \epsilon_{k l m} \partial_{l} E_{m}=-B_{k}, \quad \partial_{k} B_{k}=0, \\
& \epsilon_{k l m} \partial_{l} H_{m}=D_{k}, \quad \partial_{k} D_{k}=0, \\
& D_{k}=\varepsilon E_{k}=\varepsilon_{0} E_{k}+P_{k}, \quad B_{k}=\mu_{0} \delta I_{l}, \\
& P_{k}=\chi E_{k}, \quad H_{k}^{\prime}=H_{k}-\epsilon_{k l m} u_{l} D_{m},
\end{aligned}
$$

where $\epsilon_{k l m}$ - the Ricci tensor, $\delta_{k l}$ - the Kronecker delta tensor, $B_{k}$ - the magnetic induction vector, $D_{k}$ - the electric induction vector, $H_{k}$ - the magnetic field intensity vector, $\varepsilon$ - the permittivity constant, $\chi-$ the dielectric susceptibility.

Linearization method for the equation of electrodynamics and electric force consists in assuming that the continuum is placed in additional electric-molecular field $E$ which is time constant and space-homogeneous. This field may appear due to point defects which can be created in $\mathrm{ABCO}_{4}$ lattice during the crystal growth process. The electromagnetic processes vary in time and space the values involved being small with reference to the constant values. It is assumed that those transient values are small of the second order, that is their products are negligible.

$$
\boldsymbol{E}(\boldsymbol{x}, t)=\boldsymbol{E}+\boldsymbol{e}(\boldsymbol{x}, t), \quad \boldsymbol{H}(\boldsymbol{x}, t)=h(\boldsymbol{x}, t), \quad|\boldsymbol{e}| \ll|\boldsymbol{E}| .
$$

The value of the molecular-electric field $\boldsymbol{E}$ plays the role of a constant coefficient. The linearization (2.3) performed leads to the conclusion

where

$$
\rho \ddot{u}_{k}=C_{k l s p} \partial_{s} \partial_{l} u_{p}+\xi_{k l s p} \partial_{s} \partial_{l} u_{p}
$$

$$
\xi_{k s l p}=\chi E_{n} E_{l} \epsilon_{k s r} \epsilon_{n p r} .
$$

Let us assume, for illustration, harmonic plane waves in the form

$$
U_{p}=u_{p}^{0} \exp [\mathrm{i}(\boldsymbol{q} r-\omega t)] .
$$

On substituting (2.5) into (2.4), we obtain Christoffel's equation

$$
\left\{\left[\left(C_{k l s p}+\xi_{k l s p}\right) q_{s} q_{l}\right]-\rho v^{2}\right\} u_{p}^{0}=0,
$$

where

$\left[\left(C_{k l s p}+\xi_{k l s p}\right) q_{s} q_{l}\right]=\Lambda_{k p}^{\prime}-$ the extended acoustic tensor.

Here $q_{s}, q_{l}$ are the direction cosines of $k$.

Equation (2.6) is equivalent to dispersion equation in the form

$$
\left.\left[\left(C_{k l s p}+\xi_{k l s p}\right) q_{s} q_{l}\right]-\rho v^{2}\right]=0 .
$$

Equation (2.7) is the generalization of the classical dispersion equation. 


\section{Discussion}

Using the model with wave-linear approximation the elastic properties of the crystals belonging to the family of compounds with the general formula $\mathrm{ABCO}_{4}$ can be discussed. According to this model the elastic properties of the crystals possessing tetragonal structure can be affected in some directions by the existence of the point defects and structural imperfections $[6,7]$. These defects can create additional electric field $\boldsymbol{E}$ and influence the elastic properties of the crystal. The results obtained quite recently for $\mathrm{SrLaGaO}_{4}$ single crystals from Brillouin scattering study [8] seem to confirm our theoretical model.

Equation (2.7) is the generalization of the classical dispersion Eq. (2) presented in [8]. Let us consider two special cases

$$
\begin{array}{ll}
\boldsymbol{E}\left(E_{1}, E_{2}, E_{3}\right), & q[1,0,0], \\
\boldsymbol{E}\left(E_{1}, E_{2}, E_{3}\right), & q[1,0,0] .
\end{array}
$$

Therefore, our model belongs to the cases described by Eqs. (2.7) in the form

$$
\begin{array}{lll}
{\left[\left(C_{3113}-\chi E_{1}^{2}\right)-\rho v^{2}\right]=0,} & q[1,0,0], & \boldsymbol{u}[0,0,1], \\
{\left[\left(C_{3223}-\chi E_{2}^{2}\right)-\rho v^{2}\right]=0,} & q[0,1,0], & \boldsymbol{u}[0,0,1] .
\end{array}
$$

Equations (2.9) of transverse phonons are comparable to the results reported in paper [8] (specified by No. 12 and No. 10 in Table I).

By using the results given in Eqs. (2.9) and the experimental results No. 12 and No. 10 in Table I of paper [8] and the conclusion

$$
\rho v_{(10)}^{2}>\rho v_{(12)}^{2}
$$

we can deduce that

$$
\left|E_{1}\right|>\left|E_{2}\right| \text {. }
$$

This remark is an illustration to this theory. The relation between components of the molecular electric field $E$ (2.11) is decisive in the problem considered, for the existence of electroelastic coupling. Therefore, the anisotropy of the elastic properties of $\mathrm{SrLaGaO}_{4}$ crystals caused by the point defects created in this crystal during the growth process can be explained with the help of the dispersion equation.

\section{Conclusion}

Using theoretical model with wave-linear approximation the anisotropy of the elastic properties observed for $\mathrm{SrLaGaO}_{4}$ single crystals can be explained.

\section{Acknowledlgments}

This work was partially supported by the Research Project of Poznań University of Technology BW 21-790/96.

\section{References}

[1] M. Mukaida, S. Miyazawa, Appl. Phys. Lett. 67, 999 (1993).

[2] R. Sobolewski, P. Gierławski, W. Kula, S. Zarembiński, S.J. Lewandowski, M. Berkowski, A. Pajączkowska, B.P. Gorshunov, D.B. Lyudmirski, O.I. Sirotyński, IEEE Trans. Magn. 27, 876 (1991). 
[3] T. Hoffmann, J. Techn. Phys. 30, 165 (1989).

[4] J. Weyssenhoff, An Outline of Electromagnetics and Classical Optics, Vol. I, PWN, Warszawa 1957 (in Polish).

[5] W.K. Panofsky, M. Phylips, Classical Electricity and Magnetism, North-Holland, Amsterdam 1963.

[6] A. Pajączkowska, P. Byszewski, J. Cryst. Growth 128, 694 (1993).

[7] A. Gloubokov, R. Jabłoński, W. Ryba-Romanowski, J. Sass, A. Pajączkowska, R. Uecker, P. Reiche, J. Cryst. Growth 147, 123 (1995).

[8] M. Drozdowski, A. Pajączkowska, D. Kasprowicz, P. Ziobrowski, A. Kolos, Cryst. Res. Technol. 31, 361 (1996). 